Check for updates

Cite this: RSC Adv., 2018, 8, 34397

Received 21st May 2018

Accepted 21st September 2018

DOI: $10.1039 / \mathrm{c} 8 \mathrm{ra0} 4300 \mathrm{k}$

rsc.li/rsc-advances

\section{Synthesis of a benzyl-grafted alginate derivative and its effect on the colloidal stability of nanosized titanium dioxide aqueous suspensions for Pickering emulsions}

\author{
Meixi Feng, ${ }^{a}$ Chuanhai Gu, ${ }^{a}$ Chaoling Bao, ${ }^{a}$ Xiuqiong Chen, ${ }^{a}$ Huiqiong Yan, ${ }^{\star a b}$ \\ Zaifeng Shi, ${ }^{a}$ Xiaohong $\mathrm{Liu}^{\mathrm{a}}$ and Qiang Lin (D)*ab
}

$\mathrm{TiO}_{2}$ nanoparticles (nano- $\mathrm{TiO}_{2}$ ) as one of the most extensively used nanoscale materials easily undergo spontaneous aggregation and gravity sedimentation ascribed to their high adsorption energy, which significantly restricts their actual applications. For this reason, a benzyl-grafted alginate derivative (BAD) with good colloidal interface activity, prepared by a bimolecular nucleophilic substitution $\left(\mathrm{S}_{\mathrm{N}} 2\right)$ reaction, was used as the dispersant to stabilize nano- $\mathrm{TiO}_{2}$. The structure and colloidal properties of BAD was evaluated by FT-IR spectroscopy, ${ }^{1} \mathrm{H}$ NMR spectroscopy, thermal gravimetric analysis (TGA) and dynamic light scattering (DLS). The effects of $\mathrm{pH}$ and ionic strength on the dispersion stability of $\mathrm{BAD} / \mathrm{nano}-\mathrm{TiO}_{2}$ suspensions were also examined by DLS. To further probe its feasibility as a drug delivery system, the $\mathrm{BAD} /$ nano- $\mathrm{TiO}_{2}$ complex was applied as particulate emulsifiers to fabricate drug-loaded Pickering emulsions. Meanwhile, the morphology properties and the sustained release performance of the drugloaded Pickering emulsions were also investigated. Experimental results showed that the adsorption of BAD on nano- $\mathrm{TiO}_{2}$ was achieved by an intermolecular hydrogen bond between the carboxylic functional groups of $\mathrm{BAD}$ and the $\mathrm{Ti}-\mathrm{OH}$ of $\mathrm{TiO}_{2}$. The adsorption of $\mathrm{BAD}$ enhanced the electrostatic repulsion and steric hindrance between nano- $\mathrm{TiO}_{2}$ improving the dispersion stability of nano- $\mathrm{TiO}_{2}$ at different $\mathrm{pH}$ and ionic strength. Additionally, the obtained Pickering emulsions displayed good drug-loading capacity and sustained release performance with the release mechanism of non-Fickian transport, which exhibited great potential in the pharmaceutical field.

\section{Introduction}

$\mathrm{TiO}_{2}$ nanoparticles (nano- $\mathrm{TiO}_{2}$ ) are considered to be one of the most extensively used nanoscale materials for their unique advantages, such as good stability, high photocatalytic activity, strong UV resistance, etc. $^{\mathbf{1 , 2}}$ They have been commonly utilized as additives to commercial products such as cosmetics, pharmaceuticals, and foodstuffs. ${ }^{3-5}$ However, nano- $\mathrm{TiO}_{2}$ in suspension readily show a strong tendency to spontaneously agglomerate and then sediment due to their strong hydrophilicity, large specific surface area, and high adsorption energy, which significantly restrict their actual application in the manufacture of commercial products. ${ }^{6,7}$

\footnotetext{
${ }^{a}$ Key Laboratory of Water Pollution Treatment \& Resource Reuse of Hainan Province, College of Chemistry and Chemical Engineering, Hainan Normal University, Haikou 571158, Hainan, P. R. China. E-mail: yanhqedu@163.com; linqianggroup@163. com; Fax: +86898 66187313; Tel: +8689866275138

${ }^{b}$ Key Laboratory of Tropical Medicinal Plant Chemistry of Ministry of Education, College of Chemistry and Chemical Engineering, Hainan Normal University, Haikou 571158, Hainan, P. R. China
}

The aggregation of aqueous nano- $\mathrm{TiO}_{2}$ suspensions is owing to the London-van der Waals attractive forces between the nano$\mathrm{TiO}_{2}$ of the dispersed phase, which can be overcome by means of electrostatic repulsions between similarly charged particles arising from solvation of adsorbed layers, or most frequently by an electrostatic mechanism with the use of dispersants. ${ }^{\mathbf{6}, \mathbf{8}}$ Currently, most of reports have focused on the ways to stabilize $\mathrm{TiO}_{2}$ particles against aggregation by using surfactants or polymers as dispersants..$^{9-13}$ The rationale underlying this approach is that the adsorption of dispersants on the nanoparticles surfaces not only affects the electrostatic interactions, but also introduces additional steric interactions, which can prevent the nanoparticles surfaces from coming into direct contact. $^{\mathbf{1 4}}$

For examples, Tkachenko et al. ${ }^{15}$ studied the influence of concentration of nonionic TRITON X-100 and anionic ATLAS G3300 surfactants on the size and zeta potential of $\mathrm{TiO}_{2}$ particles in the water suspensions. The results showed that anionic ATLAS G-3300 surfactant was more effective stabilizator of aqueous suspensions of titanium dioxide, than nonionic surfactants of TRITON X-100. And it was also found that 
hydrophobic interaction had important role in the processes of stabilization of suspensions. Yang et al. ${ }^{13}$ investigated the effect of anionic sodium dodecyl sulfate monomer and micelles on the stability of aqueous dispersions of nano- $\mathrm{TiO}_{2}$ using dynamic light scattering (DLS) techniques. They found the strong steric repulsion generated by sodium dodecyl sulfate micelles can effectively prevent the aggregation and sedimentation of nano$\mathrm{TiO}_{2}$. Liufu et $a .^{\mathbf{1 6}}$ applied the poly (acrylic acid) (PAA) to stabilize the suspension of nano- $\mathrm{TiO}_{2}$, and the results showed that the stabilization of $\mathrm{PAA} /$ nano- $^{-\mathrm{TiO}_{2}}$ suspension was achieved through electrostatic repulsion, and the adsorption of PAA on the $\mathrm{TiO}_{2}$ surface may take place by hydrogen bonding and chemical interaction between the nanoparticles and the carboxyl groups of the polymer. From the above examples, we can find that the dispersant may either possess colloidal interface activity or be flexible polyelectrolytes. The employed polymer dispersants should be capable of hydrogen bonding with the metal oxide surface. ${ }^{17,18}$ And the carboxylic functional groups are often used to anchor polymeric chains to oxide nanoparticles surfaces. ${ }^{19,20}$ Although the addition of different surfactants and synthetic polymers can effectively improve the dispersion stability of nano- $\mathrm{TiO}_{2}$, the extensive use of them is bound to cause some pollution to the environment. ${ }^{21}$ Therefore, it is necessary to seek a green alternative to reduce environmental damages and environmental consequences. To note, alginate, as a natural anionic polyelectrolyte, has abundant carboxylic functional groups and other merits, such as source availability, non-toxicity, biocompatibility and biodegradability. ${ }^{22-24}$ It is these characteristics that possibly endow it with the potential to become a green dispersant for the stabilization of nano- $\mathrm{TiO}_{2}$ aqueous suspension.

Alginate, derived from marine algae and various bacteria, as a natural anionic unbranched biopolymer is a heteropolysaccharide comprised of (1-4)-linked $\beta$-D-mannuronic acid and $\alpha$-L-guluronic acid blocks distributed in an irregular blockwise pattern. ${ }^{25,26}$ As alginate molecular chain contains large amounts of free hydroxyl and carboxyl groups, intramolecular hydrogen bonds are easily formed within the molecules. $^{27,28}$ These intramolecular hydrogen bonds lead to highly stretched rigid structure of the alginate chain in aqueous solution, which is not conducive to the adsorption of alginate as a dispersant on the surface of nano- $\mathrm{TiO}_{2}$. In this case, the chemical modification of alginate by hydrophobic groups may be an effective method to enhance its molecular flexibility or colloidal interface activity, ${ }^{29,30}$ thus improving the ability to disperse nano- $\mathrm{TiO}_{2}$. As a result of available hydroxyl and carboxyl groups, chemical modification of alginate could be achieved by the acetylation, phosphorylation, sulfation, oxidation, esterification and amidation. ${ }^{27}$ Most of the modifications need the catalyst to activate the hydroxyl or carboxyl groups attributed to the rigidity of alginate chain, ${ }^{29,30}$ but they often exhibited low chemical activity. Recently, Pawar et al. reported a strategy to dissolve tetrabutylammonium (TBA) salts of alginic acid in polar aprotic solvents containing tetrabutylammonium fluoride (TBAF) for synthesis of carboxyl-modified alginate esters. This strategy makes the esterification of TBA-alginate be performed under homogeneous conditions via bimolecular substitution nucleophilic $\left(\mathrm{S}_{\mathrm{N}} 2\right)$ reactions without the aid of the catalyst, resulting in the high chemical activity with the total degree of substitution (DS) close to $1.0 .^{31,32}$ In spite of high chemical activity for $\mathrm{S}_{\mathrm{N}} 2$ reaction, the chemical characterization and colloidal properties of the corresponding alginate derivative synthesized by $S_{N} 2$ reaction is still scarce, especially when the prepared alginate derivative was used as a dispersant for the dispersion of nano- $\mathrm{TiO}_{2}$ in aqueous media.

In the present study, the benzyl-grafted alginate derivative (BAD) was prepared by $\mathrm{S}_{\mathrm{N}} 2$ reaction to improve the colloidal interface activity of alginate. To retain partial carboxylic functional groups of alginate that could be used to self-assemble into core-shell architectures and anchor polymeric chains to nano- $\mathrm{TiO}_{2}$ 's surfaces, the molar ratio of benzyl bromide and uronic acid of TBA-alginate was fixed at 50\%. The structure and colloidal property of BAD was evaluated by FT-IR spectrometer, ${ }^{1} \mathrm{H}$ NMR spectrometer, thermal gravimetric analysis (TGA) and dynamic light scattering (DLS). And then we attempted to use BAD as the dispersant to stabilize commercial nano- $\mathrm{TiO}_{2}$ with the crystalline phase of anatase in aqueous solution. The effects of $\mathrm{pH}$ and ionic strength on the dispersion stability of BAD/ nano- $\mathrm{TiO}_{2}$ suspensions were also examined by DLS. Finally, the drug-loaded Pickering emulsions were prepared by high-speed shearing method using $\mathrm{BAD} /$ nano- $\mathrm{TiO}_{2}$ complex as the particulate emulsifiers and $\lambda$-cyhalothrin as the model pesticide to probe its feasibility as a drug delivery system. The morphology properties and the release performance of the drug-loaded Pickering emulsions were also investigated. This work combined renewable alginate with inorganic nanomaterials, aiming at paving a new path for the extensive application of nano- $\mathrm{TiO}_{2}$.

\section{Experimental procedure}

\subsection{Materials}

Titanium dioxide nanoparticles (99.8\%; $\mathrm{TiO}_{2}, 10-25 \mathrm{~nm}$, anatase type), alginic acid from brown algae (CP grade), benzyl bromide (98\%), tetrabutylammonium hydroxide (25\% in water; TBAOH), tetrabutylammonium fluoride (99\%; TBAF), $N, N$ dimethylformamide (AR grade; DMF) were purchased from Aladdin Reagent Co., Ltd., China. The organic pesticide of $\lambda$ cyhalothrin (94.6\%) were provided by Yangnong Reagent Co., Ltd., Chemical, Jiangsu, China. Other solvents, such as $\mathrm{HCl}$, $\mathrm{NaOH}, \mathrm{NaCl}$, ethanol, methanol and Nile red were also purchased from Aladdin Chemical Reagent Co., Ltd., Shanghai, China. These chemicals were analytical grade and used without further purification. All aqueous solutions were prepared with deionized water.

\subsection{Synthesis and characterization of benzyl-grafted alginate derivative}

Benzyl-grafted alginate derivative (BAD) was synthesized according to previous method with some modifications. ${ }^{31,32}$ The synthesis route, as displayed in Scheme 1, involved the neutralization of the alginic acid with $\mathrm{TBAOH}$ and its 


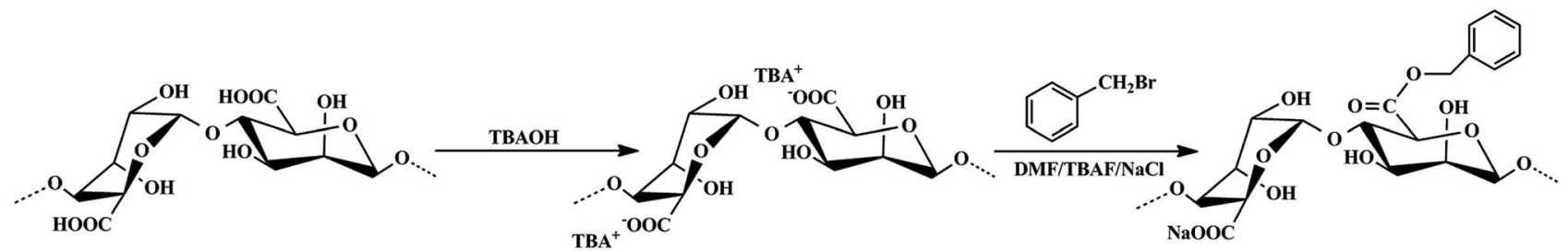

Scheme 1 The synthesis route of BAD by $S_{N} 2$ reaction.

bimolecular substitution nucleophilic $\left(\mathrm{S}_{\mathrm{N}} 2\right)$ reaction, which were detailedly described as follows.

Firstly, $4.0 \mathrm{~g}$ of alginic acid was dispersed in $100 \mathrm{~mL}$ distilled water under stirring, and the aqueous TBAOH was added dropwise until the $\mathrm{pH}$ was adjusted to 8-9. Then, the mixture was filtered under vacuum using coarse filter paper to remove any unreacted particulates. Afterward, the obtained solution was dialyzed in distilled water for 3 days and lyophilized to get the pure TBA-alginate polymer.

Subsequently, $1.5 \mathrm{~g}$ of TBA-alginate was dissolved in $100 \mathrm{~mL}$ DMF containing $1.0 \mathrm{~g}$ of TBAF and stirred vigorously to allow its complete dissolution. Then $0.19 \mathrm{~mL}$ of benzyl bromide was directly added to the TBA-alginate solution and the mixture was left to react at ambient temperature under stirring. After $24 \mathrm{~h}$ of reaction, $50 \mathrm{~mL}$ of $2.5 \mathrm{~mol} \mathrm{~L}^{-1} \mathrm{NaCl}$ aqueous solution was added to replace the existing $\mathrm{TBA}^{+}$ions with $\mathrm{Na}^{+}$ions under another $2 \mathrm{~h}$ of stirring. Finally, the resultant polymer was completely precipitated by adding ethyl acetate, then separated by centrifuge at $7000 \mathrm{rpm}$ and washed thoroughly with ethanol to remove excess reactants repeatedly. The product was dialyzed in distilled water for 3 days and lyophilized to get the pure BAD. For comparison, sodium alginate (SA) was prepared by neutralizing raw alginic acid with $\mathrm{NaOH}$ solution.

The degree of substitution (DS) for BAD during the $\mathrm{S}_{\mathrm{N}} 2$ reaction was determined by titration according to previous reports, ${ }^{32}$ which were performed by reacting alginate esters with excess $\mathrm{NaOH}$ to hydrolyze the ester groups. The chemical structure of BAD was characterized by FT-IR, ${ }^{1} \mathrm{H}$ NMR and thermogravimetric analysis. FT-IR spectra of samples were recorded on a Thermo Scientific Nicolet 6700 Fourier transform infrared spectrometer. The samples were mixed with $\mathrm{KBr}$ and compressed to semitransparent disks for spectroscopic analysis. ${ }^{1} \mathrm{H}$ NMR was performed on a Bruker AV 400 nuclear magnetic resonance spectrometer. The samples were dissolved in $\mathrm{D}_{2} \mathrm{O}$ to the concentration of $10 \mathrm{mg} \mathrm{mL}^{-1}$. Thermogravimetric analysis was carried out on a TA Q600 Thermal Analyzer in $\mathrm{N}_{2}$ at a heating rate of $20^{\circ} \mathrm{C} \mathrm{min}^{-1}$ to examine the thermal properties of the samples. Fluorescence measurement was performed on a Hitachi F7000 fluorescence spectrophotometer, which is an efficient way to detect the colloidal interface activity of BAD. Pyrene as a fluorescence probe was excited at $335 \mathrm{~nm}$ and the emission spectrum was collected in the range of $335-600 \mathrm{~nm}$ at an integration time of $1 \mathrm{~s}$ with the slit width of $2.5 \mathrm{~nm}$. The size and zeta potential of BAD micelle-like self-aggregates were measured by DLS with a Malvern Nano-ZS90 Zetasizer (UK) at a scattering angle of $90^{\circ}$ at $25^{\circ} \mathrm{C}$, employing an (He-Ne) argon laser $(\lambda=633 \mathrm{~nm})$. The polymeric micelle-like self-aggregates solution was prepared at $60{ }^{\circ} \mathrm{C}$ and diluted to the concentration of $1.0 \mathrm{mg} \mathrm{mL}^{-1}$ to avoid multiple scattering.

\subsection{Preparation and evaluation of $\mathrm{BAD} /$ nano- $^{-\mathrm{TiO}_{2}}$ suspensions}

The $\mathrm{BAD} / \mathrm{nano}^{-\mathrm{TiO}_{2}}$ suspensions were prepared by the mechanical mixing method. Briefly, $5 \mathrm{mg}$ of BAD and $10 \mathrm{mg}$ of nano- $\mathrm{TiO}_{2}$ were ultrasonically dispersed in $10 \mathrm{~mL}$ deionized water. Then the dispersions were shaken on a shaker overnight to promote the adsorption of $\mathrm{BAD}$ on nano- $\mathrm{TiO}_{2}$, thus preparing the $\mathrm{BAD} / \mathrm{nano}^{-\mathrm{TiO}_{2}}$ suspensions.

The effects of $\mathrm{pH}$ and ionic strength on the dispersion stability of $\mathrm{BAD} / \mathrm{nano}^{-\mathrm{TiO}_{2}}$ suspensions were examined by DLS, which were expressed by the change of their size and zeta potential. The $\mathrm{pH}$ effect test was performed by adjusting $\mathrm{pH}$ of the suspensions from 3 to 9 using $0.1 \mathrm{~mol} \mathrm{~L}^{-1} \mathrm{HCl}$ solution and $0.1 \mathrm{~mol} \mathrm{~L}^{-1} \mathrm{NaOH}$ solution. Similarly, the ionic strength effect test was performed by adjusting ionic strength of the suspensions to $50,100,150,200$ and $300 \mathrm{mmol} \mathrm{L}^{-1}$ by the addition of $\mathrm{NaCl}$.

To further confirm the morphology and structure of $\mathrm{BAD} /$ nano- $\mathrm{TiO}_{2}$ suspensions and the interactions between $\mathrm{BAD}$ and nano- $\mathrm{TiO}_{2}$, the scanning electron microscopy (SEM) observation, transmission electron microscope (TEM) observation and FT-IR analysis were conducted for the freeze-dried BAD/nano$\mathrm{TiO}_{2}$ suspensions. The SEM measurement was performed on a Hitachi S-3000N scanning electron microscope (Japan) after gold coating, while the TEM measurement was carried out using a JEM 2100 TEM (JEOL Co., Japan) at an acceleration voltage of $200 \mathrm{kV}$.

\subsection{Fabrication of Pickering emulsions by $\mathrm{BAD} /$ nano-TiO $_{2}$ suspensions}

The Pickering emulsions with an oil/water ratio of $1: 9(\mathrm{v} / \mathrm{v})$ were prepared by high-speed shearing method with a high shear homogenizer (T10 basic ULTRA-TURRAX, IKA, Germany), using $\mathrm{BAD} /$ nano- $^{-\mathrm{TiO}_{2}}$ complex as the particulate emulsifiers and toluene as the oil phase, as shown in Scheme 2. Detailedly, $1.0 \mathrm{~mL}$ of toluene was added to $9.0 \mathrm{~mL}$ of $\mathrm{BAD} / \mathrm{nano}^{-\mathrm{TiO}_{2}}$ suspensions with the concentrations of $5.0 \mathrm{mg} \mathrm{mL}^{-1}$ for nano$\mathrm{TiO}_{2}$ and $2.5 \mathrm{mg} \mathrm{mL} \mathrm{m}^{-1}$ for BAD to form the emulsions with the aid of $20000 \mathrm{rpm}$ high-speed shearing.

The stability to creaming of the resultant emulsions was checked by storing them at ambient temperature for $24 \mathrm{~h}$. Since emulsion droplets had a lower density than the surrounding liquid, they moved upwards rapidly and a cream-depleted 


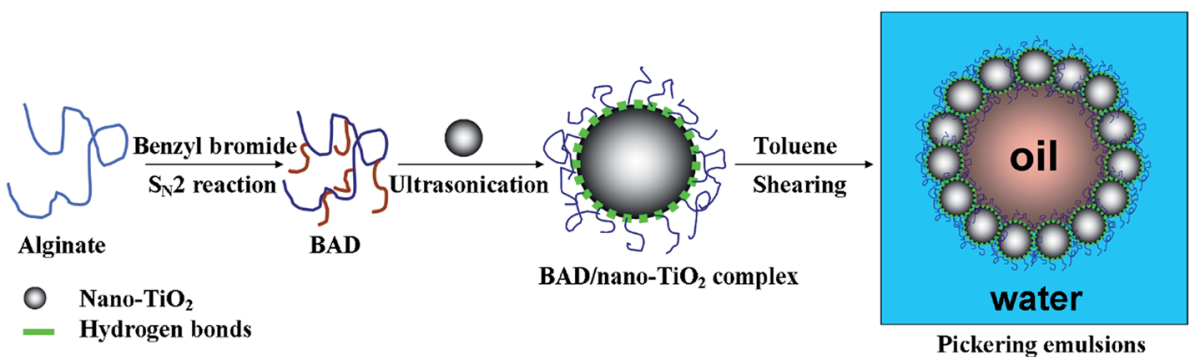

Scheme 2 Schematic illustration of the fabrication of Pickering emulsions by $\mathrm{BAD} /$ nano- $-\mathrm{TiO}_{2}$ complex.

aqueous phase was emerged at the bottom of the emulsions. To observe the microstructures of emulsion droplets, the oil phase was stained with Nile red. The emulsion droplets were placed directly onto a glass microscope slide and covered the coverslip. Then the microstructure of Pickering emulsion droplets was observed by using a Nikon Ti-S fluorescent microscope (Japan).

\subsection{Loading and release studies of $\lambda$-cyhalothrin}

$\lambda$-Cyhalothrin is a kind of broad-spectrum, highly-efficient, and long-lasting biomimetic pesticide, which can not only control various pests on crops, such as cotton, fruit trees, vegetables, and soybeans, but also control parasites on animals. For this reason, $\lambda$-cyhalothrin was chosen as the model pesticide for loading and release studies to verify the application of Pickering emulsions stabilized by $\mathrm{BAD} /$ nano- $\mathrm{TiO}_{2}$ complex as an efficient pesticide carrier. In our work, about $20 \mathrm{mg}$ of $\lambda$-cyhalothrin was fully dissolved in oil phase under stirring prior to the fabrication of Pickering emulsions. The loading of $\lambda$-cyhalothrin into Pickering emulsions was achieved based on the emulsification of $\mathrm{BAD} /$ nano- $\mathrm{TiO}_{2}$ complex.

The release of $\lambda$-cyhalothrin from the drug-loaded Pickering emulsions was performed in $50 \%$ methanol solution at ambient temperature in a stoppered conical flask. $2 \mathrm{~mL}$ of drug-loaded Pickering emulsions were enclosed into a dialysis bag (cut-off $3500 \mathrm{MW}$ ), and then the bag was immersed in $50 \mathrm{~mL}$ of $50 \%$ methanol solution. $50 \mathrm{~mL}$ of the solution was replaced with the same volume of fresh $50 \%$ methanol solution at different time intervals, which could avoid the influences of the saturated solutions. $1 \mathrm{~mL}$ of the solution was removed at different time intervals and filtered with $0.22 \mu \mathrm{m}$ membrane for the determination of $\lambda$-cyhalothrin by GC analysis using a HP6890N Gas Chromatography (Agilent, USA). The drug release procedure was performed in triplicate to calculate the standard deviation.

\section{Results and discussion}

\subsection{Preparation and characterization of BAD}

Alginic acid can be easily neutralized by TBAOH to form TBAalginate that is only partially soluble in organic solvents such as DMF and DMSO. The solubility of TBA-alginate in DMF could be significantly improved in the presence of a small amount of TBAF, which achieves the $\mathrm{S}_{\mathrm{N}} 2$ reaction for the synthesis of BAD in a homogeneous solution. ${ }^{31,32}$ To keep partial carboxylic functional groups of alginate that would be applied to adsorb nano- $\mathrm{TiO}_{2}$, benzyl bromide as the hydrophobic modifier was added at $50 \%$ stoichiometry to react with TBA-alginate. Due to the presence of $\mathrm{p}-\pi$ conjugate effect, the benzyl group has high stability and strong activity. The degree of substitution (DS) of the resultant $\mathrm{BAD}$ reached about $46.3 \%$, revealing high reactivity and reaction rate for $\mathrm{S}_{\mathrm{N}} 2$ reaction, which consisted with previous report by Pawar et al. ${ }^{32}$

FT-IR and ${ }^{1} \mathrm{H}$ NMR analyses are useful methods for elucidating the specific functional groups and structure of synthetic BAD. The FT-IR spectra of SA and BAD were displayed in Fig. 1. From the FT-IR spectrum of SA (Fig. 1a), the characteristic peaks at $2928.88,1092.95$ and $1031.74 \mathrm{~cm}^{-1}$ were respectively assigned to $\mathrm{C}-\mathrm{H}$ and $\mathrm{C}-\mathrm{O}-\mathrm{C}$ stretching vibrations of the saccharide structure. ${ }^{30}$ The peaks at 1620.13, 622.22 and $1417.40 \mathrm{~cm}^{-1}$ were attributed to asymmetric and symmetric stretching vibrations of -COO- ${ }^{33}$ In comparison with SA, BAD exhibited the similar FT-IR spectrum as shown in Fig. 1b. However, BAD showed the additional new peak at $1742.79 \mathrm{~cm}^{-1}$ for the presence of ester bonds, indicating the successful esterification of alginate by benzyl groups. Additionally, the peak of O-H at $3000-4000 \mathrm{~cm}^{-1}$ in the spectrum of BAD became sharp. The results indicated that the grafting of hydrophobic benzyl groups could destroy the original intramolecular hydrogen bonding of alginate, which could enhance its molecular flexibility. ${ }^{33-35}$

The successful synthesis of BAD was further proved by ${ }^{1} \mathrm{H}$ NMR. As shown in Fig. 2, the proton peaks of SA and BAD were marked in ${ }^{1} \mathrm{H}$ NMR spectra. The proton peaks ranging from 5.0

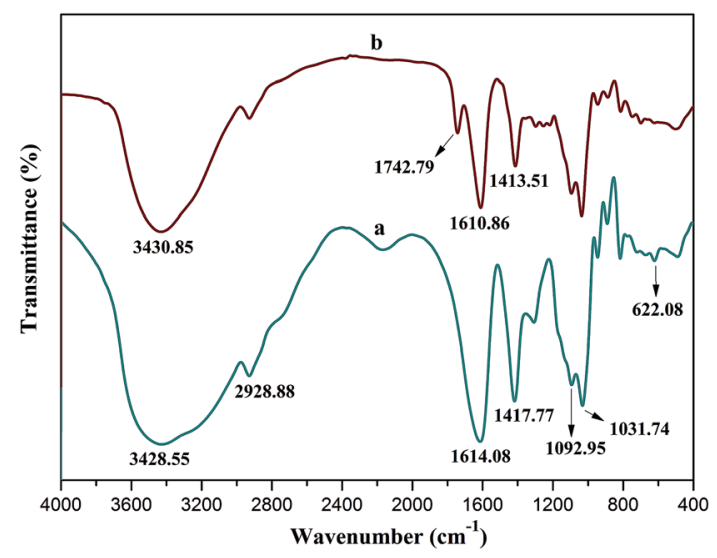

Fig. 1 FT-IR spectra of (a) SA and (b) BAD. 


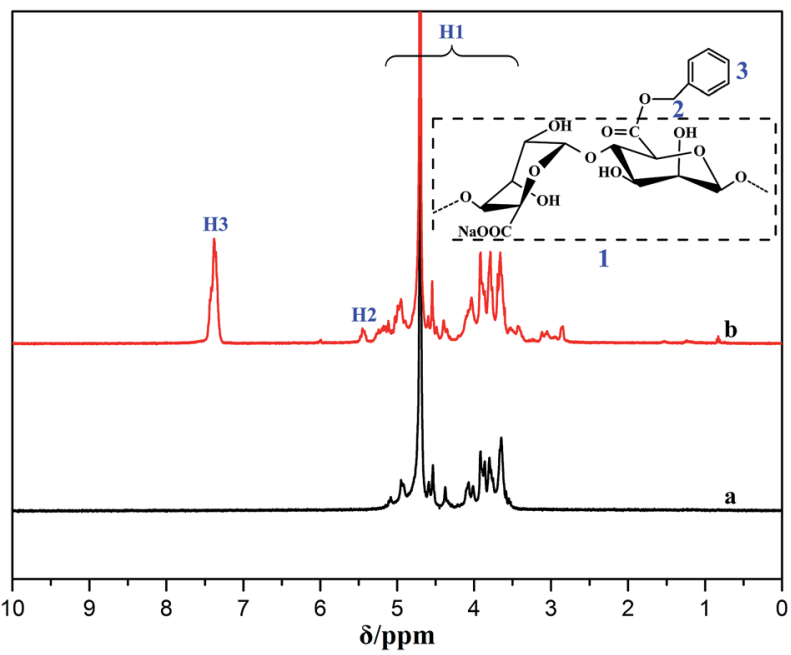

Fig. $2{ }^{1} \mathrm{H}$ NMR spectra of (a) SA and (b) BAD.

to $3.5 \mathrm{ppm}$ were ascribed to the $\mathrm{H}$ of native alginate chains. ${ }^{30} \mathrm{By}$ contrast, the spectrum of BAD showed additional new proton peaks at 7.35 and $5.45 \mathrm{ppm}$, which were respectively assigned to benzyl aromatic and benzyl $-\mathrm{CH}_{2}-$ signals. $^{32}$ The protons assignment of the new functional groups were further confirm the successful grafting of hydrophobic benzyl groups onto alginate main chains.

Thermogravimetric analysis is the most effective method for characterizing the thermal stability of polymer materials. ${ }^{36}$ The thermal gravity analysis (TGA) and derivative thermograms (DTG) for SA and BAD were shown in Fig. 3. The weight retentions of SA and $\mathrm{BAD}$ at $800{ }^{\circ} \mathrm{C}$ were respectively $30 \%$ and $24 \%$ (Fig. 3a and b), and their DTG curves displayed two main mass loss stages, which was ascribed to the losses of physically adsorbed water at low temperature $\left(80-120{ }^{\circ} \mathrm{C}\right)^{37}$ and the decomposition of polymer moieties at high temperature (200$\left.250{ }^{\circ} \mathrm{C}\right) .^{38}$ The thermal decomposition of polymer moieties resulted in the formation of $\mathrm{CO}, \mathrm{CO}_{2}$ and $\mathrm{H}_{2} \mathrm{O}$, resulting in the rapid decrease in weight. ${ }^{39}$ To note, the initiating decomposition temperature of $227^{\circ} \mathrm{C}$ for BAD was obviously smaller than

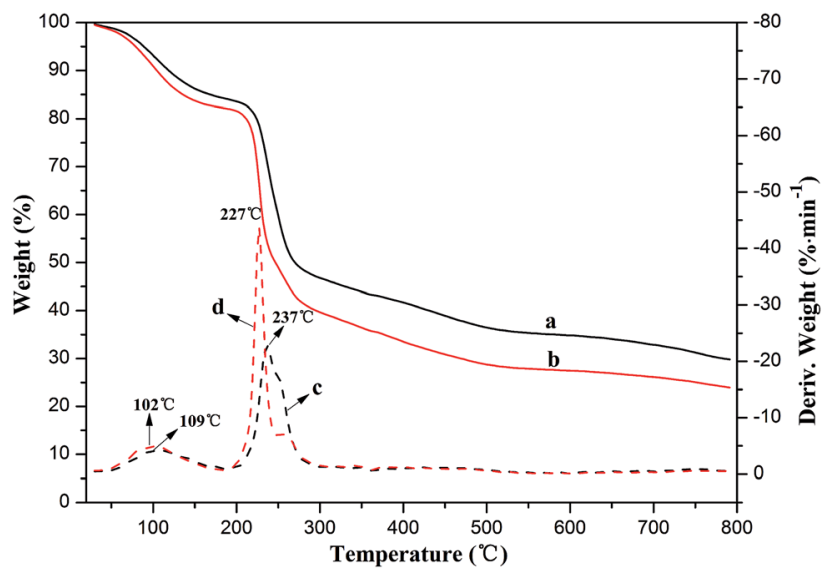

Fig. 3 Thermal gravity curves of (a) SA and (b) BAD, and derivative thermograms of (c) SA and (d) BAD. that of $237{ }^{\circ} \mathrm{C}$ for SA, indicating that BAD had lower thermal stability. As the benzyl groups was covalently bonded to the carboxyl groups of alginate during the $\mathrm{S}_{\mathrm{N}} 2$ reaction, the carboxyl groups of BAD was reduced. And the decrease of carboxyl moieties may easily resulted in the destruction of the polymeric intramolecular hydrogen molecule, thus causing the reduction of its initiating decomposition temperature. These results further implied the esterification of alginate by $\mathrm{S}_{\mathrm{N}} 2$ reaction was feasible.

Owing to the partial grafting of hydrophobic benzyl groups onto the main chains of alginate molecule, which could destroy the original intramolecular hydrogen bonding to enhance its molecular flexibility, the BAD may exhibit certain colloidal interface activity. The colloidal interface activity could be indirectly examined with the fluorescence measurement using hydrophobic pyrene as the fluorescent probe. ${ }^{37}$ There are five electronic vibration peaks in the pyrene fluorescence spectroscopy, and the ratio value of the first peak at $372 \mathrm{~nm}\left(I_{1}\right)$ to the third peak at $383 \mathrm{~nm}\left(I_{3}\right)$ is related to the micro-environmental polarity surrounding pyrene molecules, which decreases with the reduction of the micro-environmental polarity. ${ }^{36,37}$ When the $\mathrm{BAD}$ molecules aggregated to form micelle-like selfaggregates due to the hydrophobic association of benzyl groups, the hydrophobic pyrene can be encapsulated in the hydrophobic microdomains formed by benzyl groups, thus resulting the significant decrease of the ratio value of $I_{1} / I_{3}$. As shown in Fig. 4, the $I_{1} / I_{3}$ value decreased with the increase of BAD concentration. The corresponding onset concentration is the critical aggregation concentration (CAC) of BAD, which is the lowest concentration of micelle-like self-aggregates by hydrophobic association. The BAD exhibited relative low CAC value of $0.50 \mathrm{~g} \mathrm{~L}^{-1}$ in comparison with that of alginate $(1.90 \mathrm{~g}$ $\mathrm{L}^{-1}$ ), indicating good amphipathy for BAD. Therefore, it is the amphiphilic property of AAD that could achieve the adsorption of $\mathrm{BAD}$ at the oil/water interface. ${ }^{30}$

The colloidal property of BAD was further investigated by DLS with the results showed in Fig. 5. As can be seen from Fig. 5a, the BAD exhibited a narrow size distribution involving

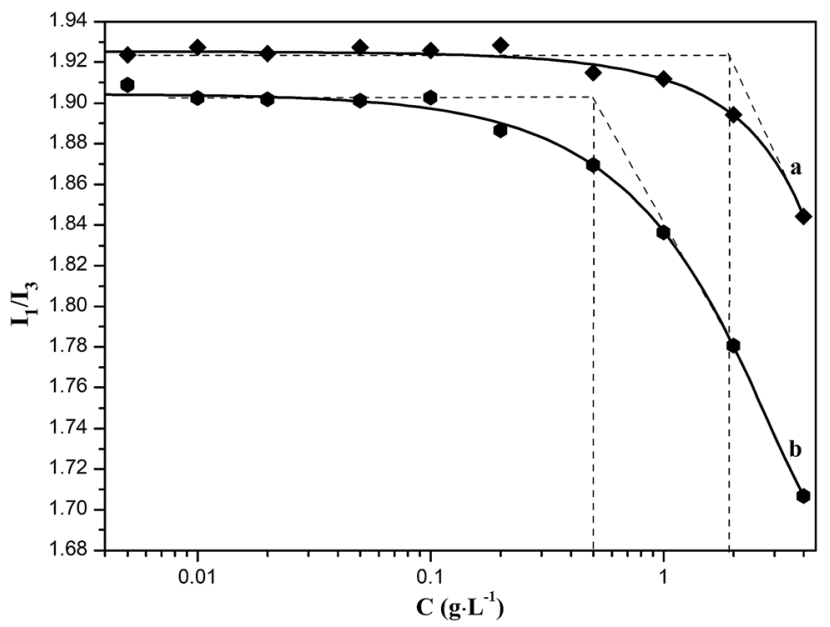

Fig. 4 Plots of pyrene fluorescence intensity $I_{1} / I_{3} v s$. the concentration of (a) sodium alginate and (b) BAD solutions. 
(a)

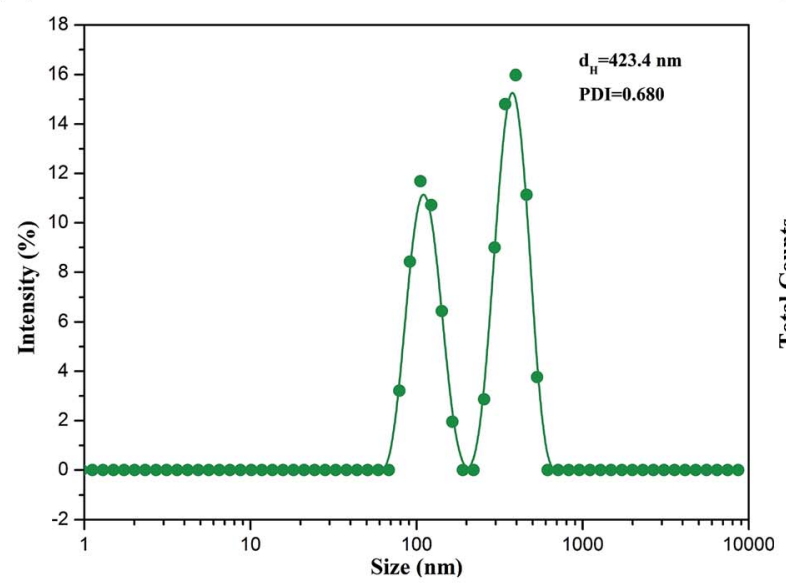

(b)

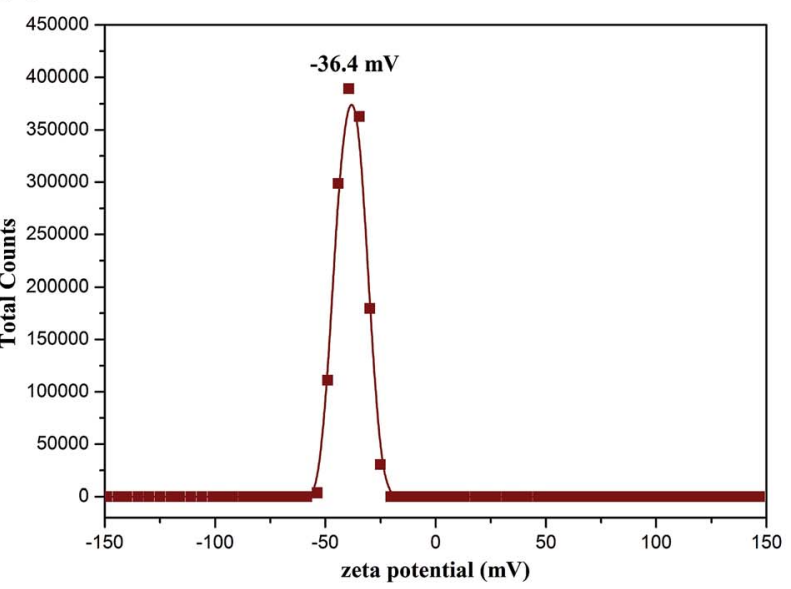

Fig. 5 Size distribution (a) and zeta potential distribution (b) of BAD micelle-like self-aggregates.

two continuous ranges, which may be ascribed to the unequal grafting of hydrophobic benzyl groups onto the alginate main chains or the degradation of molecular chains during the $\mathrm{S}_{\mathrm{N}} 2$ reaction. Their average hydrodynamic diameter was $423.4 \mathrm{~nm}$ with the PDI of 0.68. The results indicated that due to the hydrophobic association of benzyl groups, the BAD molecules aggregate to form micelle-like self-aggregates. ${ }^{37}$ This results further validated that the molecular flexibility of BAD was enhanced by hydrophobic association. ${ }^{34}$ In addition, the BAD micelle-like self-aggregates showed a relatively high negative zeta potential at about $-36.4 \mathrm{mV}$ as a result of the presence of the residual free-curling carboxyl groups (Fig. 5b). This result indicated the BAD molecules, containing both the hydrophilic main chains and hydrophobic side groups, could self-assemble into core-shell architectures, where the hydrophilic domains were exposed. It was generally considered to be stable in aqueous solution when the absolute value of zeta potential was higher than $30 \mathrm{mV}$, as the strong repulsion forces prevented the aggregation among the micelle-like self-aggregates. ${ }^{40}$ On the basis of the above results, the BAD displayed good colloidal interface activity.

\subsection{Effect of BAD on the colloidal stability of nano- $\mathrm{TiO}_{2}$}

As the BAD possessed good colloidal interface activity, it can be utilized to regulate the colloidal properties of nano- $\mathrm{TiO}_{2}$ through electro-sterical repulsion..$^{15}$ The method to achieve the dispersion stability of nano- $\mathrm{TiO}_{2}$ is that the adsorption of BAD on the nano- $\mathrm{TiO}_{2}$ 's surfaces via carboxylic functional groups to prevent the nanoparticles' surfaces from coming into direct contact. ${ }^{14}$ Since the change of $\mathrm{pH}$ and ionic strength could influence the charge density and size of BAD, the adsorption of polymer onto the surfaces of nanoparticles depends notably on the $\mathrm{pH}$ and ionic strength of the solution. ${ }^{16}$

3.2.1 Effect of $\mathbf{p H}$ on the zeta potential and size of $\mathrm{BAD} /$ nano- $\mathrm{TiO}_{2}$ suspensions. Commercial nano-TiO ${ }_{2}$ with the crystalline phase of anatase contain a large amount of $\mathrm{Ti}-\mathrm{OH}$ on their surfaces, and they will show different chargeabilities ascribed to the influence of the $\mathrm{pH}$ of the aqueous phase. As shown in Fig. 6a, with the increase of pH, the zeta potential of the nano- $-\mathrm{TiO}_{2}$ gradually decreased from a positive value to a negative value. Meanwhile, it could be also observed that the point of zero charge (PZC) of the nano- $\mathrm{TiO}_{2}$ suspension was at $\mathrm{pH}$ value of 5.6, which was close to the previous reports of $\mathrm{pH}$ at the $\mathrm{PZC}$ for anatase nano- $\mathrm{TiO}_{2} \cdot{ }^{41}$ When the $\mathrm{pH}$ value of the aqueous phase was less than 5.6, the surface of nano- $\mathrm{TiO}_{2}$ was positively charged attributed to the protonation of $\mathrm{Ti}-\mathrm{OH}$. But, when the $\mathrm{pH}$ of the aqueous phase was higher than 5.6, the surface of nano- $-\mathrm{TiO}_{2}$ was negatively charged owing to the deprotonation of Ti-OH. ${ }^{42}$ In the vicinity of the PZC, due to the least charge on the surface of the nano- $\mathrm{TiO}_{2}$, the electrostatic repulsion between the nanoparticles was the weakest. So the agglomeration phenomenon between the particles was the most significant, making nano-TiO ${ }_{2}$ suspension exhibit a higher average hydrodynamic diameter $\left(d_{\mathrm{H}}\right)$ as shown in Fig. 6b. However, the zeta potential and $d_{\mathrm{H}}$ of BAD was less influenced by $\mathrm{pH}$ value. When $\mathrm{BAD}$, as a dispersant, was adsorbed on nano$\mathrm{TiO}_{2}, \mathrm{BAD} /$ nano-TiO ${ }_{2}$ complex exhibited strong negative charge with good colloidal properties, and their zeta potentials were lower than $-30 \mathrm{mV}$, which was favorable for reducing the aggregation of nano- $\mathrm{TiO}_{2}$, thus improving their dispersion stability. As we can see from the inset, the pictures of BAD/nano$\mathrm{TiO}_{2}$ suspensions at various $\mathrm{pH}$ exhibited good dispersion stability. It was the higher electrostatic and steric effects of BAD that made the average $d_{\mathrm{H}}$ of $\mathrm{BAD} /$ nano- $^{-\mathrm{TiO}_{2}}$ complex significantly smaller than that of the single $\mathrm{TiO}_{2}$ nanoparticle. ${ }^{14}$

3.2.2 Effect of ionic strength on the zeta potential and size of $\mathbf{B A D} /$ nano- $\mathrm{TiO}_{2}$ suspensions. The effect of ionic strength on the dispersion stability of $\mathrm{BAD} / \mathrm{nano}^{-\mathrm{TiO}_{2}}$ suspensions was evaluated in the $\mathrm{NaCl}$ solution. There was an ionotropic effect existed in the aqueous $\mathrm{NaCl}$ solution, which could induce the electrostatic shielding effects to reduce the chargeability of the charged nanoparticles. ${ }^{43}$ As shown in Fig. 7a, with the increase of ionic strength, the zeta potential of nano- $-\mathrm{TiO}_{2}$ and $\mathrm{BAD} /$ nano- $\mathrm{TiO}_{2}$ complex gradually increased because of the counter ion $\mathrm{Na}^{+}$that aggregated around the negatively charged $\mathrm{Ti}-\mathrm{O}^{-}$ and carboxyl groups. When BAD was adsorbed on nano- $\mathrm{TiO}_{2}$, their electrostatic shielding effect in $\mathrm{NaCl}$ solution was 
(a)

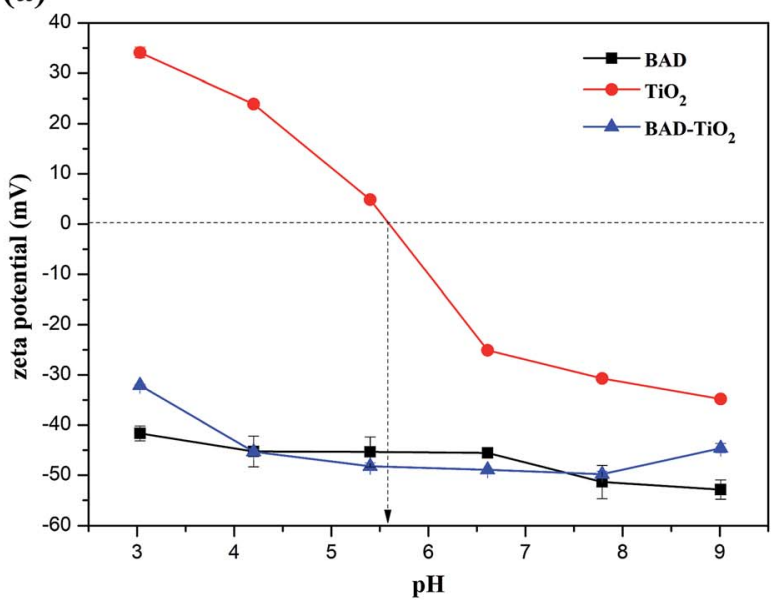

(b)

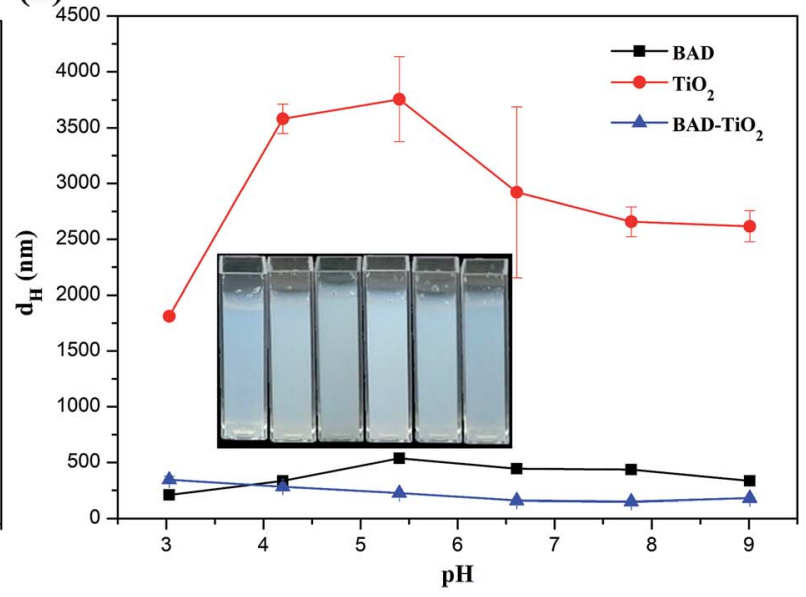

Fig. 6 Effects of $\mathrm{pH}$ on (a) zeta potential and (b) size of aqueous $\mathrm{BAD}$, nano- $\mathrm{TiO}_{2}$ and $\mathrm{BAD} /$ nano- $-\mathrm{TiO}_{2}$ suspensions (inset: the pictures of $\mathrm{BAD}$ / nano- $\mathrm{TiO}_{2}$ suspensions at various $\mathrm{pH}$ ).

significantly weakened. Moreover, as shown in the inset (Fig. 7b), the adsorption of BAD greatly reduces the agglomeration behavior of nano-TiO ${ }_{2}$ in different ionic strength aqueous phases, resulting in a significant decrease in hydrodynamic diameter. As reported, salt can not only reduce the surface potential of the colloidal particles, but also cause their coagulation/flocculation, which is attributed to the electrostatic contribution to the interparticle interactions. ${ }^{44}$ With the gradual increase of ionic strength, their $d_{\mathrm{H}}$ showed a trend of first increasing and then decreasing, resulted from the existence of certain coagulation/flocculation effects.

Although the higher electrostatic and steric effects of BAD could significantly decrease the average $d_{\mathrm{H}}$ of $\mathrm{BAD} / \mathrm{nano}^{-\mathrm{TiO}_{2}}$ complex in comparison to the nano- $\mathrm{TiO}_{2}$, the $d_{\mathrm{H}}$ of $\mathrm{BAD} /$ nano$\mathrm{TiO}_{2}$ complex was much higher than the expected individual nano- $\mathrm{TiO}_{2}(10-25 \mathrm{~nm})$. The results perhaps could be explained by the fact that nano- $\mathrm{TiO}_{2}$ were too small to achieve the dispersion stability of individual nano- $\mathrm{TiO}_{2}$ due to the high adsorption energy between nanoparticles. Additionally, a hydrodynamic diameter was the actual object's diameter plus a strongly bound water shell generated by the continuous phase and the BAD on the surface of nano-TiO ${ }_{2}{ }^{37}$ So the $\mathrm{BAD} /$ nano$\mathrm{TiO}_{2}$ complex revealed higher $d_{\mathrm{H}}$ than the actual size of the individual nano- $\mathrm{TiO}_{2}$.

3.2.3 Morphology and FT-IR analysis of $\mathrm{BAD} /$ nano-TiO $_{2}$ complex. The morphology of the freeze-dried $\mathrm{BAD} / \mathrm{nano}^{-\mathrm{TiO}_{2}}$ suspensions was evaluated by SEM observation. As shown in Fig. $8 \mathrm{a}$, the single nano- $\mathrm{TiO}_{2}$ were easily agglomerated because of the lack of interfacial activity and gravity sedimentation of nano- $\mathrm{TiO}_{2}$, which may severely restrict its actual applications. However, the adsorption of BAD on the surface of nano- $\mathrm{TiO}_{2}$ significantly improved the dispersibility of the nano- $\mathrm{TiO}_{2}$, and the BAD film could be observed between the dried nano- $\mathrm{TiO}_{2}$, as shown in Fig. 8b. Therefore, the adsorption of BAD enhanced the steric hindrance between nano- $\mathrm{TiO}_{2}$ to make them stably dispersive. (a)

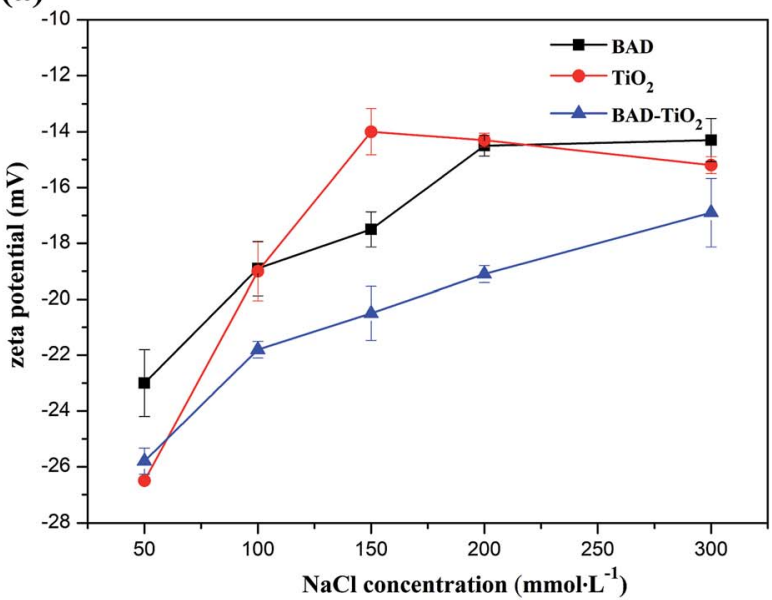

(b)

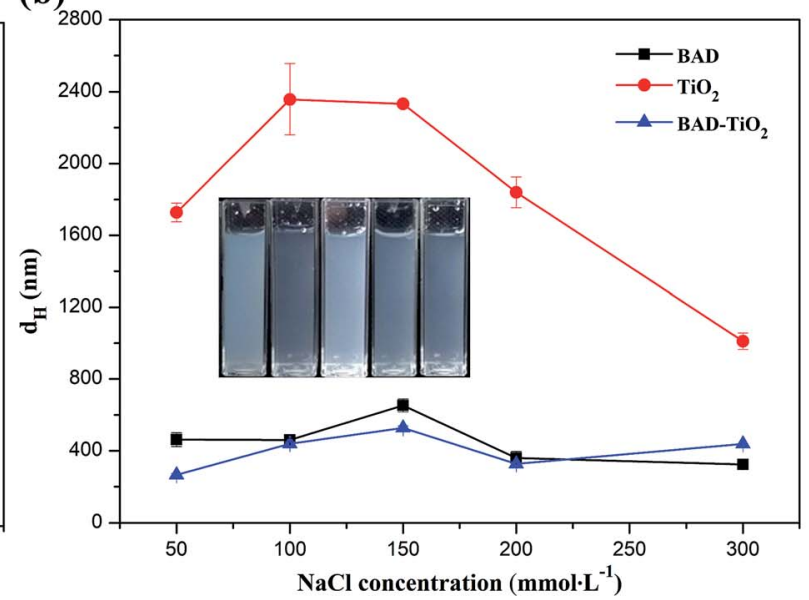

Fig. 7 Effects of ionic strength on (a) zeta potential and (b) size of aqueous $\mathrm{BAD}$, nano- $-\mathrm{TiO}_{2}$ and $\mathrm{BAD} /$ nano- $\mathrm{TiO}_{2}$ suspensions (inset: the pictures of $\mathrm{BAD} /$ nano- $-\mathrm{TiO}_{2}$ suspensions at various ionic strength). 
The structure of $\mathrm{BAD} /$ nano- $\mathrm{TiO}_{2}$ complex was also examined by TEM measurement. As we can see from Fig. 9, most of nano$\mathrm{TiO}_{2}$ aggregated to form a cluster of nanoparticles with the size of $140 \mathrm{~nm}$, which were in the middle of a micelle-like structure, whereas the $\mathrm{BAD}$ was in the corona areas. As the zeta potential of $\mathrm{BAD} /$ nano-TiO $_{2}$ complex was almost constant with the change of the $\mathrm{pH}$ values, the hydrophilic domain of BAD could be still exposed outside, ${ }^{45}$ i.e., the cluster of nano- $\mathrm{TiO}_{2}$ was in the hydrophobic core of micelle-like self-aggregates. These results showed that nano- $\mathrm{TiO}_{2}$ can interact with benzyl group to achieve the adsorption of $\mathrm{BAD}$ on the surface of nano- $\mathrm{TiO}_{2}$.

To further investigate the interaction between $\mathrm{BAD}$ and nano- $\mathrm{TiO}_{2}$ in $\mathrm{BAD} /$ nano- $^{-\mathrm{TiO}_{2}}$ suspensions, the FT-IR measurement was performed on the freeze-dried $\mathrm{BAD} / \mathrm{nano}^{-\mathrm{TiO}_{2}}$

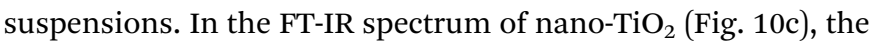
broad peak at $3442.08 \mathrm{~cm}^{-1}$ was assigned to the stretching vibration of $\mathrm{Ti}-\mathrm{OH}$. The characteristic peaks at 1632.65 and $650.58 \mathrm{~cm}^{-1}$ were respectively attributed to the bending and stretching vibration of Ti-O. ${ }^{16}$ In Fig. $10 \mathrm{~b}$, the $\mathrm{BAD} / \mathrm{nano}^{-\mathrm{TiO}_{2}}$ complex contained both the main characteristic diffraction peaks of nano- $\mathrm{TiO}_{2}$ and that of the BAD, in spite of the decrease of diffraction intensity, indicating that BAD was adsorbed onto the nanoparticles' surfaces by ultrasound and shaking. It was worth noting that the peaks of hydroxyl stretching in the spectrum of the $\mathrm{BAD} /$ nano- $\mathrm{TiO}_{2}$ complex became much broader after absorbing BAD in comparison with that of nano- $\mathrm{TiO}_{2}$. Meanwhile, the peaks of hydroxyl stretching at $3442.08 \mathrm{~cm}^{-1}$ for nano- $\mathrm{TiO}_{2}$ red shifted to $3431.04 \mathrm{~cm}^{-1}$, indicating the formation of the intermolecular hydrogen bond between the carboxylic functional groups of $\mathrm{BAD}$ and the $\mathrm{Ti}-\mathrm{OH}$ of nano- $\mathrm{TiO}_{2}$ in $\mathrm{BAD} /$ nano- $^{-\mathrm{TiO}_{2}}$ suspensions. $^{46,47}$
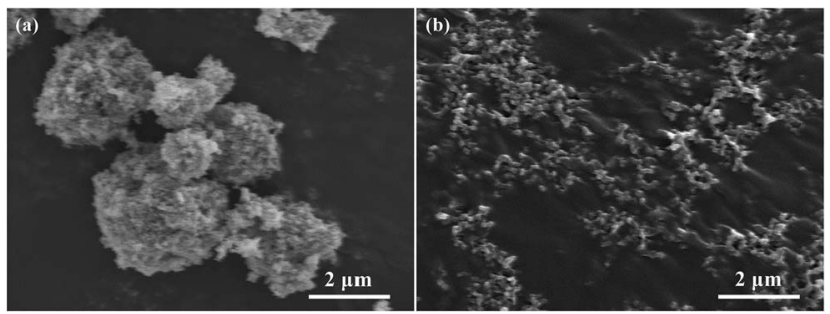

Fig. 8 SEM images of (a) $\mathrm{TiO}_{2}$ nanoparticles and (b) $\mathrm{BAD} /$ nano- $-\mathrm{TiO}_{2}$ complex.

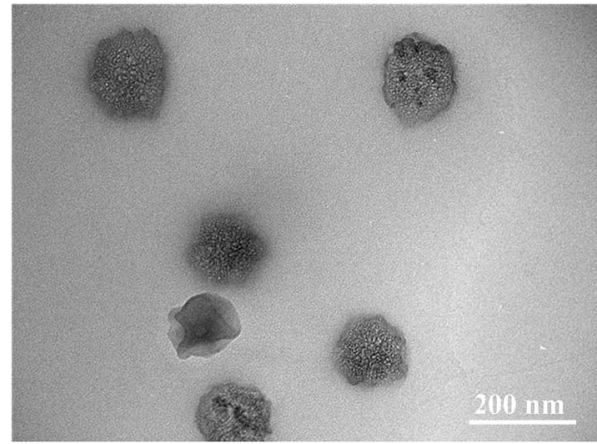

Fig. 9 TEM image of $\mathrm{BAD} /$ nano- $-\mathrm{TiO}_{2}$ complex.

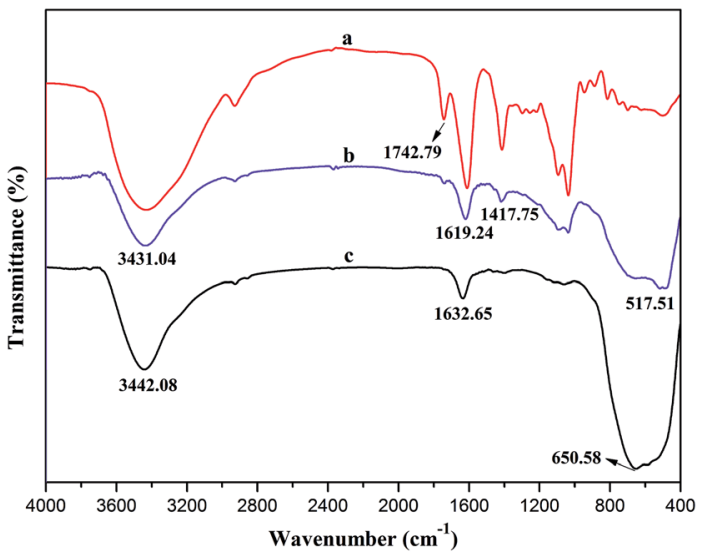

Fig. 10 FTIR spectra of (a) BAD, (b) BAD/nano- $\mathrm{TiO}_{2}$ complex and (c) $\mathrm{TiO}_{2}$ nanoparticles.

\subsection{Preparation and release studies of the drug-loaded pickering emulsions}

To further illustrate the colloidal stability of $\mathrm{BAD} /$ nano- $^{-\mathrm{TiO}_{2}}$ complex and expand their applications in pharmacology field, the drug-loaded Pickering emulsions were fabricated using $\mathrm{BAD} /$ nano- $\mathrm{TiO}_{2}$ complex as the particulate emulsifiers. As shown in Fig. 11, both of the freshly prepared Pickering emulsions stabilized by nano- $\mathrm{TiO}_{2}$ and $\mathrm{BAD} /$ nano- $\mathrm{TiO}_{2}$ complex were homogeneous and milky. After storing them at ambient temperature for $24 \mathrm{~h}$, both of the Pickering emulsions revealed emulsion phase and cream-depleted aqueous phase, owing to the upward movement of the emulsion droplets. By contrast, gravity sedimentation was emerged at the bottom of the Pickering emulsions stabilized by nano- $\mathrm{TiO}_{2}$, attributed to the poor dispersibility of single nano- $\mathrm{TiO}_{2} \cdot{ }^{48}$ And the Pickering emulsions stabilized by $\mathrm{BAD} / \mathrm{nano}^{-\mathrm{TiO}_{2}}$ complex revealed good emulsion stability.

As the oil/water ratio was fixed at $1: 9(\mathrm{v} / \mathrm{v})$ during the preparation of the Pickering emulsion, the emulsion phase would emerge at the surface of the cream-depleted aqueous phase because of its lower density. The emulsion droplets were taken from the upper emulsion phase, and their
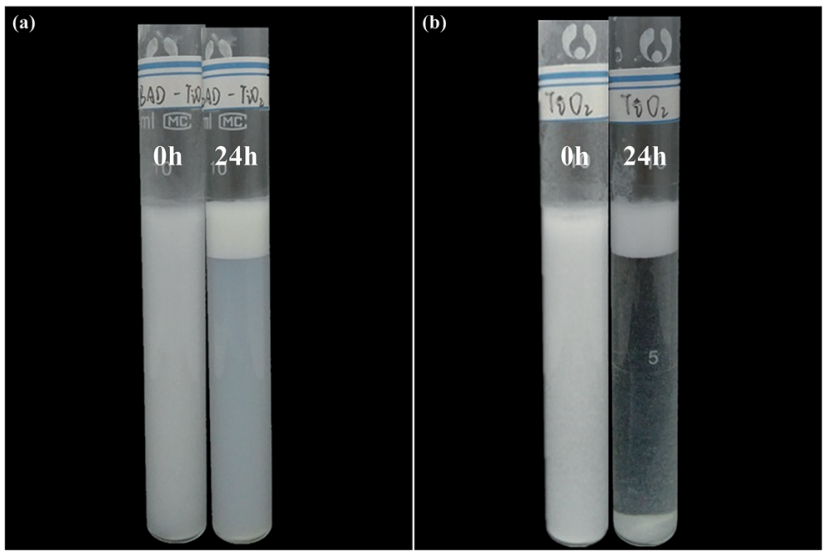

Fig. 11 Photograph of Pickering emulsions stabilized by (a) BAD/ nano- $\mathrm{TiO}_{2}$ complex and (b) $\mathrm{TiO}_{2}$ nanoparticles respectively stored at ambient temperature for $\mathrm{O} \mathrm{h}$ and $24 \mathrm{~h}$. 
microstructures were observed by fluorescent microscope after fluorescence staining. The fluorescent images of the dyed Pickering emulsions stabilized by nano- $\mathrm{TiO}_{2}$ and $\mathrm{BAD} /$ nano$\mathrm{TiO}_{2}$ complex were shown in Fig. 12. The emulsion droplets appeared red for the Nile red excitation. As oil-soluble Nile red only existed in oil phase and appeared red for its excitation, the emulsion type was confirmed to be oil-in-water type. ${ }^{49}$ The emulsion droplets stabilized by $\mathrm{BAD} / \mathrm{nano}^{-\mathrm{TiO}_{2}}$ complex exhibited good spherical shape, while the emulsion stabilized by nano- $-\mathrm{TiO}_{2}$ exhibited the coalescence phenomenon owing to the demulsification, indicating that $\mathrm{BAD} /$ nano- $-\mathrm{TiO}_{2}$ complex displayed better emulsifying performance than single nano$\mathrm{TiO}_{2}$.

The release of $\lambda$-cyhalothrin was achieved through its diffusion and the slow destruction of the drug-loaded Pickering emulsion, which was applied to indirectly analyze the emulsion stability. The release curves of $\lambda$-cyhalothrin from the drugloaded Pickering emulsions respectively stabilized by nano-

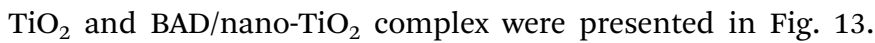
Both of the release curves showed a slow release in the whole stage. The irreversible adsorption of the particulate emulsifiers at the oil-water interface, which probably formed a mechanically robust layer may account for the slow release of $\lambda$ -
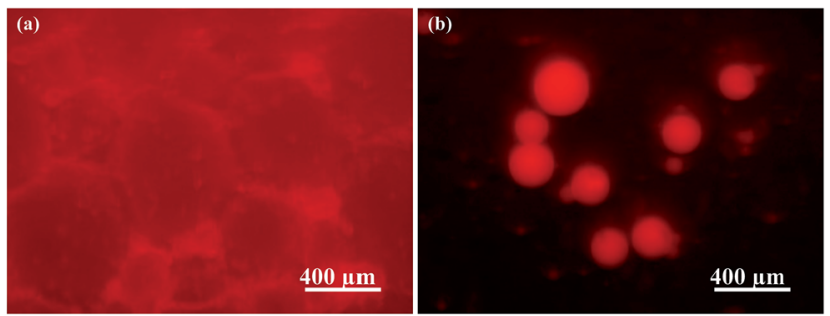

Fig. 12 Fluorescent images of the dyed Pickering emulsions respectively stabilized by (a) $\mathrm{TiO}_{2}$ nanoparticles and (b) $\mathrm{BAD} /$ nano- $-\mathrm{TiO}_{2}$ complex in dark field.

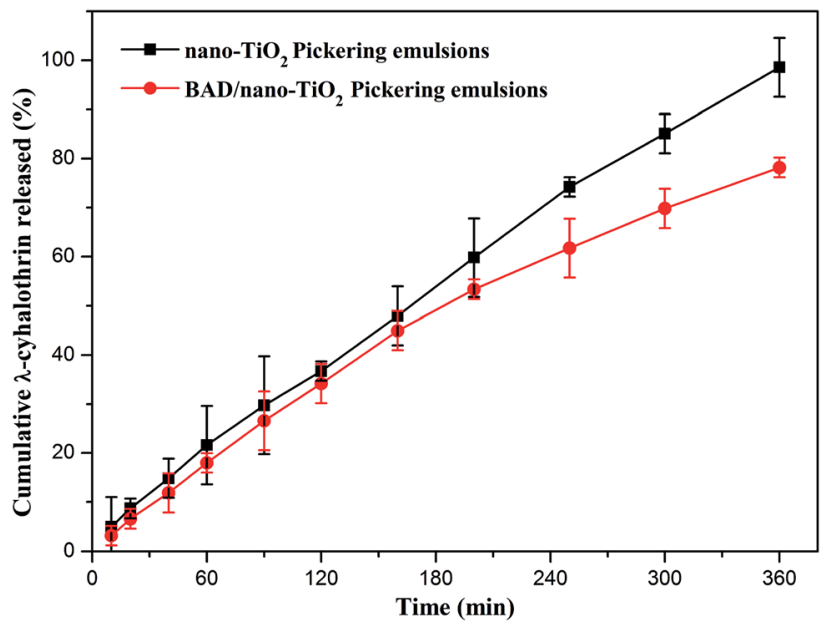

Fig. 13 Release profiles of $\lambda$-cyhalothrin from the drug-loaded Pickering emulsions respectively stabilized by $\mathrm{TiO}_{2}$ nanoparticles and BAD/ nano- $\mathrm{TiO}_{2}$ complex (error bars represent the standard deviation of three replicates). cyhalothrin. ${ }^{49}$ Within the initial 150 min, both of Pickering emulsions respectively stabilized by nano- $\mathrm{TiO}_{2}$ and $\mathrm{BAD} /$ nano$\mathrm{TiO}_{2}$ complex had the same release speed owing to the release of dissociative drug. But, after $150 \mathrm{~min}$, the drug-loaded Pickering emulsions stabilized by $\mathrm{BAD} /$ nano- $\mathrm{TiO}_{2}$ complex exhibited a slower release than the drug-loaded Pickering emulsions stabilized by nano- $\mathrm{TiO}_{2}$, attributed to the electrostatic forces and intermolecular hydrogen bonds formed between BAD that was absorbed on the surfaces of nano- $\mathrm{TiO}_{2}$, which could retard the release of $\lambda$-cyhalothrin. ${ }^{50}$ To further investigate the release mechanisms, Ritger \& Peppas model was used to analyze the release data. The model is expressed by the following equation. ${ }^{51}$

$$
M_{t} / M_{\infty}=K t^{n}
$$

where $M_{t} / M_{\infty}$ is the fractional release of drug in time $t, K$ is a constant incorporating structural and geometrical characteristics of the delivery system and $n$ is the diffusion exponent characteristic of the release mechanism. For normal Fickian diffusion the value of $n=0.5$, for case II diffusion $n=1.0$ and values of $n$ intermediate between the above limits indicate nonFickian transport.

This model was applied for analysis of the data in Fig. 13 where $M_{t} / M_{\infty} \leq 0.6$. The high correlation coefficient $(r>0.99)$ indicated that the release data could be fitted well with the model equation. The $n$ values of the drug-loaded Pickering emulsions stabilized by nano- $\mathrm{TiO}_{2}$ and the drug-loaded Pickering emulsions stabilized by $\mathrm{BAD} /$ nano- $-\mathrm{TiO}_{2}$ complex were respectively 0.8234 and 0.8989 , which ranged between 0.5 and 1.0 , indicating that the release mechanism of $\lambda$-cyhalothrin from the drug-loaded Pickering emulsions was non-Fickian diffusion transport, ${ }^{52}$ which implied that water migration into the Pickering emulsions and diffusion of the drug through the Pickering emulsions as well as the slow destruction of the Pickering emulsion were carried out simultaneously. Consequently, it is the drug-loading and sustained release properties that make the Pickering emulsions stabilized by $\mathrm{BAD} / \mathrm{nano}^{-\mathrm{TiO}_{2}}$ complex possess great potentials to become the good candidates for their use in pharmaceutical applications.

\section{Conclusions}

In summary, the benzyl-grafted alginate derivative (BAD) was successfully prepared by $\mathrm{S}_{\mathrm{N}} 2$ reaction to improve its colloidal interface activity. Afterwards, the $\mathrm{BAD}$, as the dispersant, was adsorbed on the surfaces of commercial nano- $\mathrm{TiO}_{2}$ by the mechanical mixing method. Due to the good colloidal interface activity, the $\mathrm{BAD}$ not only made $\mathrm{BAD} /$ nano- $\mathrm{TiO}_{2}$ complex possess strong negative charge to enhance the electrostatic repulsions among the nano- $\mathrm{TiO}_{2}$, but also weakened the electrostatic shielding effect in $\mathrm{NaCl}$ solution to reduce the agglomeration of nano- $\mathrm{TiO}_{2}$. It was the adsorption of $\mathrm{BAD}$ that enhanced the electrostatic repulsion and steric hindrance between nano- $\mathrm{TiO}_{2}$ to improve the dispersion stability of nano$\mathrm{TiO}_{2}$ at different $\mathrm{pH}$ and ionic strength. Moreover, the adsorption of $\mathrm{BAD}$ on nano- $\mathrm{TiO}_{2}$ was achieved by the intermolecular 
hydrogen bond between the carboxylic functional groups of $\mathrm{BAD}$ and the Ti-OH of nano-TiO ${ }_{2}$. The resultant $\mathrm{BAD} /$ nano-TiO $_{2}$ complex was used as the particulate emulsifiers to stabilize the drug-loaded Pickering emulsions. The obtained Pickering emulsions exhibited good drug-loading capacity and sustained release performance with the release mechanism of nonFickian transport, which may have great potentials as the carrier for drug delivery.

\section{Conflicts of interest}

There are no conflicts to declare.

\section{Acknowledgements}

This work was financially supported by the Natural Science Foundation of Hainan Province (218QN233) and the National Natural Science Foundation of China (21566009) and the Innovation and Scientific Research Projects for Graduates of Hainan Province.

\section{Notes and references}

1 H. Xu, S. Ouyang, L. Liu, P. Reunchan, N. Umezawaace and J. Ye, J. Mater. Chem. A, 2014, 2, 12642-12661.

2 S. Farrokhpay, Adv. Colloid Interface Sci., 2009, 151, 24-32.

3 C. O. Robichaud, A. E. Uyar, M. R. Darby, L. G. Zucker and M. R. Wiesner, Environ. Sci. Technol., 2009, 43, 4227-4233.

4 S. H. Joo, S. R. Al-abed and T. Luxton, Environ. Sci. Technol., 2009, 43, 4954-4959.

5 M. Erhayem and M. Sohn, Sci. Total Environ., 2014, 468-469, 249-257.

6 S. Fazio, J. Guzmán, M. T. Colomer, A. Salomoni and R. Moreno, J. Eur. Ceram. Soc., 2008, 28, 2171-2176.

7 S. Elbasuney, Appl. Surf. Sci., 2017, 409, 438-447.

8 E. Chibowski, L. Holysz, K. Terpilowski and A. E. Wiacek, Croat. Chem. Acta, 2007, 80, 395-403.

9 A. E. Wiacek, E. Anitowska, A. V. Delgado, L. Hołysz and E. Chibowski, Colloids Surf., A, 2014, 440, 110-115.

10 I. S. Bouhaik, P. Leroy, P. Ollivier, M. Azaroual and L. Mercury, J. Colloid Interface Sci., 2013, 406, 75-85.

11 J. P. Holmberg, E. Ahlberg, J. Bergenholtz, M. Hassellov and Z. Abbas, J. Colloid Interface Sci., 2013, 407, 168-176.

12 N. Veronovski, P. Andreozzi, C. La Mesa and M. SfiligojSmole, Surf. Coat. Technol., 2010, 204, 1445-1451.

13 Y. J. Yang, A. V. Kelkar, X. Zhu, G. Bai, H. T. Ng, D. S. Corti and E. I. Franses, J. Colloid Interface Sci., 2015, 450, 434-445.

14 Y. Lu, S. Zhou and L. Wu, J. Dispersion Sci. Technol., 2011, 33, 497-505.

15 N. H. Tkachenko, Z. M. Yaremko, C. Bellmann and M. M. Soltys, J. Colloid Interface Sci., 2006, 299, 686-695.

16 S. Liufu, H. Xiao and Y. Li, J. Colloid Interface Sci., 2005, 281, 155-163.

17 X. Yu and P. Somasundaran, J. Colloid Interface Sci., 1996, 177, 283-287.

18 S. Mathur and B. M. Moudgil, J. Colloid Interface Sci., 1997, 196, 92-98.
19 K. K. Das and P. Somasundaran, Colloids Surf., A, 2001, 182, 25-33.

20 M. A. Neouze and U. Schubert, Monatsh. Chem., 2008, 139, 183-195.

21 I. Kalashnikova, H. Bizot, B. Cathala and I. Capron, Langmuir, 2011, 27, 7471-7479.

22 M. George and T. E. Abraham, J. Controlled Release, 2006, 114, 1-14.

23 C. M. Silva, A. J. Ribeiro, M. Figueiredo, D. Ferreira and F. Veiga, AAPS J., 2006, 7, 903-913.

24 S. B. Hua, H. Z. Ma, X. Li, H. X. Yang and A. Q. Wang, Int. J. Biol. Macromol., 2010, 46, 517-523.

25 F. Vallée, C. Müller, A. Durand, S. Schimchowitsch, E. Dellacherie, C. Kelche, J. C. Cassel and M. Leonard, Carbohydr. Res., 2009, 344, 223-228.

$26 \mathrm{H}$. Bu, A. L. Kjøniksen, A. Elgsaeter and B. Nyström, Colloids Surf., A, 2006, 278, 166-174.

27 S. N. Pawar and K. J. Edgar, Biomaterials, 2012, 33, 32793305.

28 W. Mackie, S. Perez, R. Rizzo, F. Taravel and M. Vignon, Int. J. Biol. Macromol., 1983, 5, 329-341.

29 L. Q. Yang, B. F. Zhang, L. Q. Wen, Q. Y. Liang and L. M. Zhang, Carbohydr. Polym., 2007, 68, 218-225.

30 J. S. Yang, H. B. Ren and Y. J. Xie, Biomacromolecules, 2011, 12, 2982-2987.

31 S. N. Pawar and K. J. Edgar, Biomacromolecules, 2011, 12, 4095-4103.

32 S. N. Pawar and K. J. Edgar, Carbohydr. Polym., 2013, 98, 1288-1296.

33 M. S. Islam and M. R. Karim, Colloids Surf., A, 2010, 366, 135140.

34 H. R. Nie, A. H. He, J. F. Zheng, S. S. Xu, J. X. Li and C. C. Han, Biomacromolecules, 2008, 9, 1362-1365.

35 X. Chen, H. Yan, W. Sun, Y. Feng, J. Li, Q. Lin, Z. Shi and X. Wang, Polym. Bull., 2015, 72, 3097-3117.

36 M. Liu, L. Dai, H. Shi, S. Xiong and C. Zhou, Mater. Sci. Eng. C, 2015, 49, 700-712.

37 H. Yan, X. Chen, J. Li, Y. Feng, Z. Shi, X. Wang and Q. Lin, Carbohydr. Polym., 2016, 136, 757-763.

38 R. Moriana, F. Vilaplana and M. Ek, Carbohydr. Polym., 2016, 139, 139-149.

39 Z. Qin, L. Ji, X. Yin, L. Zhu, Q. Lin and J. Qin, Carbohydr. Polym., 2014, 101, 947-953.

40 Y. An, M. Chen, Q. Xue and W. Liu, J. Colloid Interface Sci., 2007, 311, 507-513.

41 M. Zhu, H. T. Wang, A. A. Keller, T. Wang and F. T. Li, Sci. Total Environ., 2014, 487, 375-380.

42 H. He, Y. Cheng, C. Yang, G. Zeng, C. Zhu and Z. Yan, J. Environ. Sci., 2017, 54, 135-141.

43 C. Yi, Y. Yang, Y. Zhu, N. Liu, X. Liu, J. Luo and M. Jiang, Langmuir, 2012, 28, 9211-9222.

44 T. Phan-Xuan, A. Thuresson, M. Skepö, A. Labrador, R. Bordes and A. Matic, Cellulose, 2016, 23, 3653-3663.

45 F. Loosli, L. Vitorazi, J. F. Berret and S. Stoll, Water Res., 2015, 80, 139-148.

46 H. B. Deng, X. Y. Li, B. Ding, Y. M. Du, G. X. Li, J. H. Yang and X. W. Hu, Carbohydr. Polym., 2011, 83, 973-978. 
47 D. Kong, G. Lu, M. Wu, Z. Shi and Q. Lin, ACS Sustainable Chem. Eng., 2017, 5, 3465-3470.

48 N. Elek, R. Hoffman, U. Raviv, R. Resh, I. Ishaaya and S. Magdassi, Colloids Surf., A, 2010, 372, 66-72.

49 Z. Hu, S. Ballinger, R. Pelton and E. D. Cranston, J. Colloid Interface Sci., 2015, 439, 139-148.
50 B. D. Kevadiya, G. V. Joshi and H. C. Bajaj, Int. J. Pharm., 2010, 388, 280-286.

51 P. L. Ritger and N. A. Peppas, J. Controlled Release, 1987, 5, 23-36.

52 H. Kaygusuz and F. B. Erim, React. Funct. Polym., 2013, 73, 1420-1425. 